Type 2 diabetes and insulin resistance among euglycaemic and diabetic patients with

\title{
Pulmonary Tuberculosis in Saudi Arabia
}

Authors: Dr. Fareed H. Almaleki, confident@ hotmail.co.uk

\section{Institutions}

1. Saudi Arabia Ministry of Health

${ }^{2}$. Tuberculosis and Chest Diseases Centre in Jeddah

${ }^{3}$ Liverpool School of Tropical Medicine, Liverpool, United Kingdom.

\section{Running title:}

Key words: tuberculosis, type 2 diabetes mellitus, Insulin resistance, adults, Kingdom of Saudi Arabia. Corresponding author:

Funding: This research was funded by the Saudi Arabian Ministry of Health. 


\section{ABSTRACT}

Background: Patients with Tuberculosis (TB) are more likely to have Type 2 Diabetes Mellitus (T2DM). Individuals with insulin resistance (IR) with normal glucose may also be at risk for TB, but there are no data available.

Methods: To explore whether T2DM and IR are risk factors for TB we enrolled patients receiving TB treatment in Saudi Arabia and healthy controls to assess the frequency of both conditions in both populations. Controls were adults attending the TB clinics to obtain a screening certificate for labour reasons. All participants were screened for fasting plasma glucose (FPG), insulin, glycated haemoglobin (HbA1c) and lipids. We assessed the severity of the clinical presentation of TB among patients with T2DM, IR and normal glucose.

Results: 175 adults with pulmonary TB (cases) and 140 controls were recruited. Fifty-one (29\%) of TB cases had T2DM and 27 (22\%) normoglycemic IR compared to 39 (27.9\%) and 26 (25.7\%) of the controls, respectively. Six (3.3\%) of cases and 12 (8.6\%) of controls were unaware of their T2DM diagnosis. TB was associated with pre-diabetes (AOR 5.112, $\mathrm{p}=0.032$ ), low level of risky HDL Cholesterol (AOR 0.316, $\mathrm{p}=0.001$ ) and non-Saudi nationality (AOR 4.018, $\mathrm{p}<0.001$ ). Cases were more likely to eat fast foods and to have a poor diet than controls $(p<0.001)$.

Conclusion: TB cases were more likely to be of non-Saudi nationality, to be male and to have T2DM and prediabetes, but were not more likely to have IR than controls.

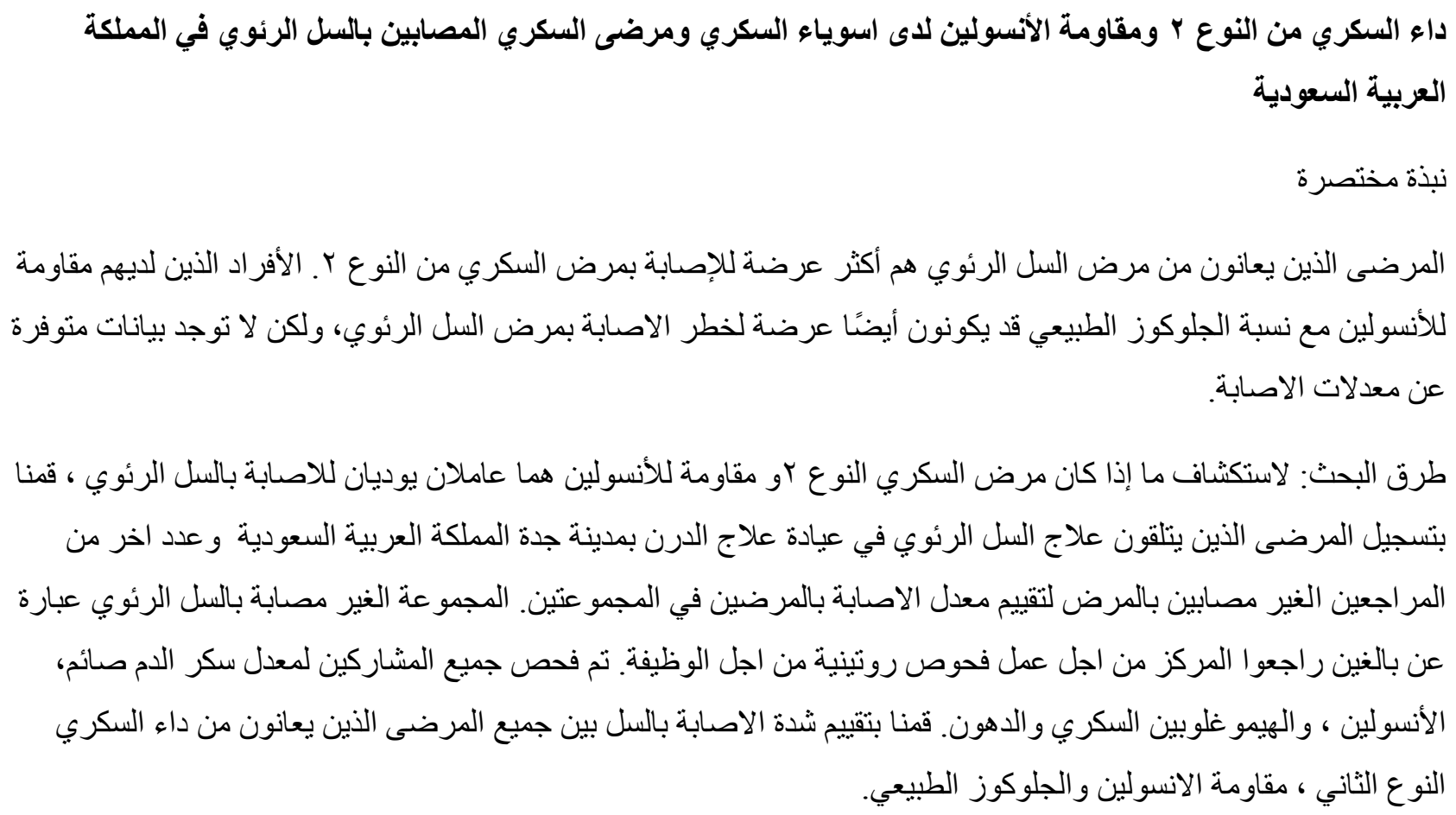




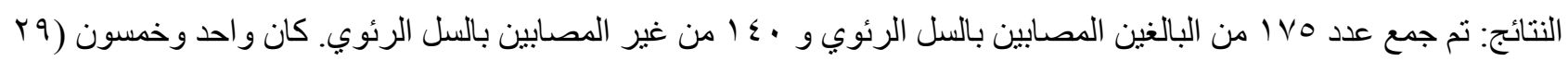

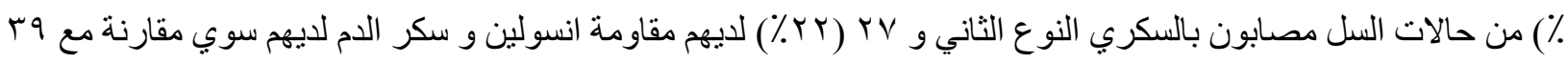

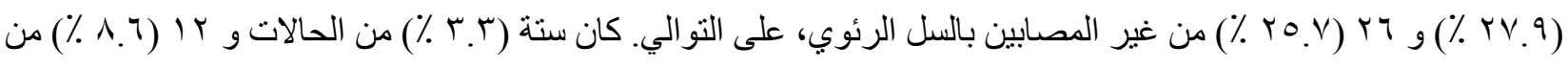
غير المصابين بالسل الرئوي غير مدركين انهم مصابون بالسكري النوع الثاني. وارتبط السل مع ما قبل السكري ( ، (P = 0.032 ، و انخفاض مستوى كوليسترول البروتين الدهني عالي الكثافة ( AOR 0.316 ،

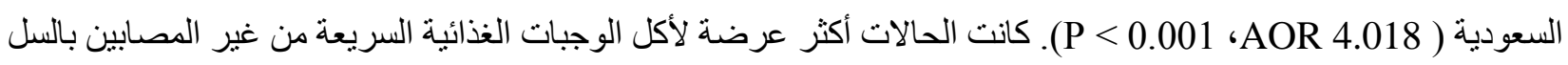

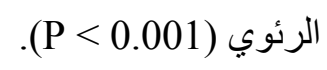

الخلاصة: كانت حالات السل الرئوي اكثر في غير السعوديين ، وترتفع غيهم الاصابة بمرض السكري النوع الثاني وما قبل مرض الن السكري ، ولكن لم يكن لديهم مقاومة انسولين أكثر من غير المصابين بالسل الرئوي. 


\section{Introduction}

Type 2 Diabetes mellitus (T2DM) is often metabolically the end result from detrimental interactions between behavioural, environmental and genetic risk factors (1). Its prevalence in the world has increased dramatically in recent decades as a result of lifestyle changes, resulting in an epidemiological shift from communicable to non-communicable diseases (2). T2DM is a syndrome resulting from reduced insulin secretion and insulin resistance (IR) associated with obesity, family history of T2DM, sedentary lifestyles and some ethnicities. Although T2DM is defined by glucose levels increased above an accepted cut off, glucose concentrations that are high but below this cutoff are also associated with some deleterious health effects, and this is known as pre-diabetes (3). T2DM is the seventh leading cause of death, affecting 422 million people in 2014. This number is expected to rise to 592 million by 2035 (4) with $80 \%$ living in LMICs where tuberculosis (TB) is prevalent $(4,5)$. Studies around the world have indicated that $12-44 \%$ of patients with TB have T2DM (6-11). T2DM enhances vulnerability TB infection, increasing the risk of TB threefold (12) as it impairs immune responses. Patients with T2DM often experience renal failure, impaired cellmediated immunity, pulmonary microangiopathy and micronutrient deficiencies which increase an individual's susceptibility to TB (12-14).

At present, there has been a drastic change over recent decades with a global re-emergence of the T2DM-TB association due to the expansion of T2DM in low and middle income countries (LMIC) (15) in areas where TB is still highly prevalent (16). There was an estimated 10 million TB incident cases in 2017, resulting in 1.6 million deaths (27), while T2DM, the seventh leading cause of death in 2016, affected 422 million in 2014, with an estimated 1.6 million deaths in 2016 (17). It is anticipated that by 2035 the number of individuals with T2DM will rise to 592 million (4) with $80 \%$ of them living in LMICs where TB is prevalent $(4,5)$. This study aimed to describe the prevalence of DM and IR in Saudi Arabia among patients with TB from Jeddah city and apparently healthy controls to investigate the association of IR with TB. 


\section{Methods}

This was a case control study including patients with bacteriologically-confirmed pulmonary TB $\geq$ 18 years old registered at the TB and Chest Diseases Centre in Jeddah city; Saudi Arabia who had responded to the intensive phase of treatment (first 2 months). Controls were individuals attending the same centre who were being treated for other pulmonary conditions or who were undergoing routine examinations for occupational requests to discard TB and who were deemed not to have TB. Both cases and controls underwent a structured interview and physical examination. Data collected consisted of general demographic characteristics, medical history and potential risk factors for T2DM and IR such as lifestyle, physical activity, diet and BMI and anthropometry.

The clinical severity of TB was assessed using a scoring system modified as described by GilSantana (2016), which included the presence or absence of each TB-related symptom (chest pain, sputum, cough, haemoptysis, fever, night sweats, poor appetite, weight loss and dyspnoea) to explore whether disease severity varied among TB patients with and without IR and/or T2DM. Data on clinical presentation at the time of diagnosis was collected from the treatment registers.

Participants provided $10 \mathrm{ml}$ of venous blood samples after fasting for 12 hours to measure HbA1c (Chemiluminescence Technology, Siemens Dimension Exl), glucose and the lipid profiles including Total Cholesterol, LDL Cholesterol, HDL Cholesterol and triglycerides on an automated chemistry analyser (Roche Cobas C601) and insulin (Roche Cobas E601) at the King Fahad Hospital laboratory in Jeddah. The automated immunoassay test analyser (ADVIA centaur xp) was used to determine the insulin level. Although the reference standard method for IR is the euglycemic clamp technique, we used the hhomeostasis model assessment-2-Insulin resistance (HOMA2-IR) calculation, as this method is more practical. The HOMA2-IR cut off point is $<2$ using the Oxford University Software HOMA2 calculator (http://www.dtu.ox.ac.uk/homacalculator/) (18).

Sample size was estimated for unmatched case-control studies with a power of $80 \%$, a ratio of 1 case per 1 control and the proportion of TB cases with T2DM being 35\% compared to $20 \%$ for controls. We estimated this required 140 cases and controls to detect an odds ratio (OR) of 2.0 at the 
$95 \%$ significance level if $10 \%$ of the population were exposed to the risk factor. A second sample size calculation was conducted for IR, as a secondary objective. We expected that the prevalence of IR was $20 \%$ in the TB cases and 5\% in the control group. To assess this difference would require a sample size of 77 participants. As this sample size was smaller, we accepted the sample size for T2DM.

Patients were classified as having normal glucose or T2DM according to their medical history, glycaemic status and HbA1c level using the WHO guidelines. IR status was classified according the Homa2-IR calculator with a cut-off point for IR $<2.0$. FPG $\geq 126 \mathrm{mg} / \mathrm{dl}$ ) $[7.0 \mathrm{mmol} / \mathrm{L}]$ or $\mathrm{HbA} 1 \mathrm{c} \geq$ $6.5 \%$ were considered abnormal. Patients with FPG $<125 \mathrm{mg} / \mathrm{dl}[6.9 \mathrm{mmol} / \mathrm{L}]$ were considered to have IR if HOMA2-IR was $\geq 2$. Participants with FPG $<110 \mathrm{mg} / \mathrm{dl}(6.1 \mathrm{mmol} / \mathrm{L}), \mathrm{HbA} 1 \mathrm{c}<6.5 \%$ and HOMA2-IR $<2$ were considered not having T2DM or IR.

We compared the characteristics of patients with and without IR using SPSS software. Univariate analysis and multiple backward stepwise logistic regression analyses were conducted to identify risk factors, presented as crude (OR) and adjusted odds ratios (AOR) with 95\% confidence intervals $(95 \% \mathrm{CI})$.

\section{Ethical considerations:}

The study obtained ethical approval from LSTM Research Ethics Committee and the Department of Preventive Medicine and Ministry of Health, Riyadh, Saudi Arabia. All participants provided written informed consent to participate.

\section{Results}

A total of 175 adult patients with confirmed pulmonary TB (cases) who had received TB treatment for at least two months and 140 controls attending the same centre were recruited. We used systematic sampling, with every $5^{\text {th }}$ patient attending the centre being invited, until the desired sample size was achieved. The demographic characteristics of the participants are shown in table 1. Cases were younger than controls with median age of 33 versus 40 years, respectively $(\mathrm{p}<0.001)$. 
One hundred eleven $(63.4 \%)$ cases and $83(59.3 \%)$ controls were male $(p=0.453)$. Cases were more likely to be single $(58,33.1 \%$ versus $33,23.6 \%, \mathrm{p}=0.012)$ and to have primary education $(47$, $26.9 \%$ versus $21,15.0 \%)$ than controls, but less likely to have intermediate $(13,7.4 \%)$ or secondary education $(33,18.9 \%)$ than controls $(32,22.9 \%$ and $36,25,7 \%$, respectively) $(p=0.001)$. Cases were more likely to work in the private sector $(34,19.4 \%)$ and to be manual workers $(46,26.3 \%)$ $(14,10.0 \%$ and $27,19.3 \%$, respectively), but less likely to work in government $(19,13.6 \%)$ or nonemployed categories $(74,52.9 \%)$ than cases $(9,5.1 \%$ and $80,45.7 \%$, respectively) $(\mathrm{p}=0.002)$. Cases were also less likely to be Saudis $((37,21.1 \%$ versus $73,52.1 \%, \mathrm{p}<0.001)$. Although frequencies were low for individual countries, large proportions were from Somalia $(27,15.4 \%)$, Yemen $(25,14.3 \%)$, Ethiopia $(17,9.7 \%)$ and Pakistan $(16,9.1 \%)$

Cases were less likely to have a family history of diabetes $(52,29.7 \%)$ than controls $(57,40.7 \%)$ (p $=0.041)$ and most relatives with $\mathrm{T} 2 \mathrm{DM}$ were first degree relatives $(48,27.4 \%$ versus $49,35.0 \%, \mathrm{p}=$ 0.148). Cases had a lower prevalence of hypertension $(9,5.1 \%$ versus $31,22.1 \%, \mathrm{p}<0.001)$ and the proportion of cases taking hypertensive medications was lower than controls $(8,4.6 \%$ versus 23 , $16.4 \%$, respectively, $\mathrm{p}<0.001)$. Cases were less likely to have hyperlipidaemia $(8,4.6 \%$ and 28 , $20.0 \%, \mathrm{p}<0.001)$ and to be taking lipid lowering therapy $(5,2.9 \%$ versus $17,12.1 \%, \mathrm{p}=0.002)$. The lifestyle and diet of cases and controls. The monthly income was < SAR 3000 (GBP 600) in $139(79.4 \%)$ cases compared to $93(66.4 \%)$ controls and only $5(2.9 \%)$ cases and $13(9.2 \%)$ controls had an income above SAR $5000(\mathrm{p}=0.010)$. Cases were more likely to report high levels of stress $(19,10.9 \%)$ than controls $(3,2.1 \%)(\mathrm{p}=0.009)$ and to have a poor diet $(48,27.4 \%$ versus $11,7.9 \%$, respectively), eating more fast foods than controls $(\mathrm{p}=0.018)$.

Male cases had lower BMI than male controls (22 versus 26, p < 0.001) and were less likely to be obese; having lower waist and hip circumference $(\mathrm{p}<0.001)$. Among females, cases had a lower BMI (22 versus $28, \mathrm{p}<0.001)$ and were less likely to be obese, had a lower median waist and hip circumference than controls $(\mathrm{p}<0.001)$. 
The metabolic and clinical characteristics of participants. Variables are presented as medians interquartile ranges (IQR) as they had skewed distributions. Cases had lower median triglycerides than controls (94 versus $106 \mathrm{mg} / \mathrm{dL}$, respectively, $\mathrm{p}=0.009$ ) with a lower proportion having high triglycerides concentrations $(8,4.6 \%$ versus $20,14.3 \%$, respectively, $\mathrm{p}=0.009)$. Cases also had higher median HDL cholesterol $(49 \mathrm{mg} / \mathrm{dL}$ and $42 \mathrm{mg} / \mathrm{dL}$, respectively, $\mathrm{p}<0.001)$ and a lower proportion had at risk HDL cholesterol levels $(60,34.3 \%$ versus $76,54.3 \%$, respectively, $\mathrm{p}<$ 0.001). Hypertension was less frequent among cases than controls, with a lower median systolic blood pressure (SBP) $(118 \mathrm{mmHg})$ than controls $(121 \mathrm{mmHg})$, although this difference is unlikely to be of clinical significance.

The diabetes status of cases and controls is shown in table 2 . The total number of diabetic patients was calculated by merging patients with known T2DM (from medical history) and FPG test results. Despite being younger, cases were more likely to be diabetic patients $(51,29.0 \%, 95 \%$ CI 22.5 $36.5 \%)$ than controls $(39,27.9 \%, 95 \%$ CI $20.6-36.1 \%)$, but this difference was not statistically significant at the univariate analysis $(\mathrm{p}=0.801)$ (figure 2). Among diabetic patients, a higher proportion of cases used insulin $(10,19.6 \%, 95 \%$ CI $9.8-33.1 \%)$ than controls $(2,5.1 \%, 95 \%$ CI $0.6-17.3 \%$ ), although the difference was not statistically significant $(\mathrm{p}=0.061)$ (figure 3 ).

Normoglycemic participants were classified according to whether they had IR using a HOMA2-IR cut-off of $\geq 2$. Out of 175 cases, 124 had a normoglycemic status $(71 \%, 95 \%$ CI $63.5-77.5 \%)$ compared to $101(72.1 \%, 95 \%$ CI $63.9-79.4 \%)$ of 140 controls. Among 124 normoglycemic cases, $27(22 \%, 95 \%$ CI $14.9-30.1 \%)$ had IR compared to 26 of 101 normoglycemic controls $(25.7 \%$, 95\% CI 17.6 - 35.4\%). There was no statistically significant difference at the univariate analysis between normoglycemic cases and controls regarding to diabetic status and IR $(p=0.485)$ (figure 4).

The total number of patients with metabolic syndrome was calculated according to the National Cholesterol Education Program Adult Treatment Panel III Guidelines (NCEP ATP III) definition. 
The proportion of patients with metabolic syndrome was lower among cases (45 (25.7\%), 95\% CI $19.4-32.9 \%)$ than controls (68 (48.6\%), 95\% CI $40.0-57.2 \%)(\mathrm{p}<0.001)$.

\section{Logistic Regression}

As many variables had significant correlations with each other, we undertook multivariable backward stepwise logistic regression for clusters of variables, to identify the main variables for each cluster. Variables with p-values $<0.2$ were selected into a multivariable backward stepwise logistic regression. These included age, nationality, marital status, education, occupation, T2DM, family history of diabetes, hypertension, takes hypertensive medication, hyperlipidaemia, takes lipid lowering therapy, polycystic ovaries, monthly income, level of stress, family overweight, number of dinner meals last week, participant self-rating the diet quality, fast food consumption, WHR, BMI, FPG, HbA1c, fasting plasma insulin, total cholesterol, triglycerides, LDL Cholesterol, HDL Cholesterol, systolic BP and diastolic BP. Multivariable backward stepwise logistic regressions were conducted separately for demographic characteristics, medical history, lifestyle and diet, anthropometry and metabolic and clinical characteristics of cases and controls. As shown in appendix (13). For each cluster, the variables remaining statistically significant were carried forward into multivariate analysis using backward statistics regressions with replacement. We adjusted for age, gender, marital status, obesity and ethnicity to obtain the Adjusted ORs (AOR).

The results of the final multivariable backward stepwise logistic regression including T2DM are shown in table 3. Cases were more likely to be non-Saudis (AOR 3.565, 95\% CI 1.83 - 6.97, p < 0.001), to have T2DM (AOR 2.950, 95\% CI $1.31-6.62, \mathrm{p}=0.009$ ) and to indicate they had a fair (AOR 12.944, 95\% CI 5.60 - 29.93, p < 0.001) or poor diet (AOR 33.625, 95\% CI 10.75 - 105.20, p $<0.001)$ than controls. Cases were more likely to eat fast foods $1-2$ times/week (AOR 2.468, 95\% CI $0.03-5.93, p=0.043$ ) or $2-3$ times/month (AOR 2.655, 95\% CI $1.00-7.04, p=0.050)$, less likely to be obese (AOR 0.190, 95\% CI $0.05-0.70, p=0.012$ ), to have higher total cholesterol (AOR 3.035, 95\% CI $1.34-6.87, \mathrm{p}=0.008)$ and a lower level of risky HDL Cholesterol (AOR $0.400,95 \%$ CI $0.21-0.76, p=0.005)$ than controls. 
The results of the final multivariable backward stepwise logistic regression including metabolic syndrome are shown in table 4. This further analysis excluded T2DM as a single factor, as this diagnosis is included in the definition of metabolic syndrome. Cases were more likely to be nonSaudis (AOR 3.448, 95\% CI $1.81-6.58, \mathrm{p}<0.001$ ) and to indicate they had a fair (AOR 12.893, 95\% CI $5.71-29.11, \mathrm{p}<0.001)$ or poor $\operatorname{diet}(\mathrm{AOR} 30.197,95 \%$ CI $10.10-90.29, \mathrm{p}<0.001)$ than controls. Cases were less likely to be obese (AOR 0.224, 95\% CI $0.06-0.81, p=0.022$ ), to have higher total cholesterol (AOR 3.382, 95\% CI 1.55 - 7.40, p = 0.002) than controls.

\section{Comparison of TB cases with and without IR and T2DM}

We conducted a sub-analysis for (a) normoglycemic TB cases without IR; (b) euglycemic TB cases with IR and (c) TB cases with T2DM to determine factors among TB cases independently associated with T2DM and IR. Independent variables for these analyses were chosen based on the literature of risk factors for T2DM. We will call normoglycemic TB cases without IR as 'TBcontrols' ( $n=97)$ and euglycemic TB cases with IR as 'TB-IR' $(n=29)$ and the 49 diabetic TB cases as 'TB-diabetic'.

The demographic characteristics of TB-diabetic and TB-IR cases and TB-controls. Among TBdiabetic cases, $40(81.6 \%)$ were male and nine (18.4\%) female compared to $57(58.8 \%)$ and 40 (41.2\%) TB-controls, respectively $(\mathrm{p}=0.006)$. TB-diabetic cases were older than TB-controls, with median age of 49 and 27 years, respectively $(\mathrm{p}<0.001)$, were more likely to be married $(39,79.6 \%$ versus $52,53.6 \%$, respectively, $\mathrm{p}<0.001)$ and to work in the private sector $(16,32.7 \%$ versus 13 , $13.4 \%)$ or as manual workers $(15,30.6 \%$ versus $26,26.8 \%)(\mathrm{p}=0.017)$. There were no significant differences between TB-IR cases and TB-controls. Given the small sample size we were unable to conduct a logistic regression analysis. 
The medical histories of TB-diabetic and TB-IR cases and TB-controls. TB-diabetic cases were more likely to have hyperlipidaemia than TB-controls $(6,12.2 \%$ versus $1,1.0 \%, \mathrm{p}=0.009)$ and to take lipid lowering therapy $(4,8.2 \%$ versus $0,0.0 \%$, respectively) $(\mathrm{p}=0.008)$. TB-IR cases in turn had a higher prevalence of heart problems than TB-controls $(2,6.9 \%$ versus $0,0.0 \%, \mathrm{p}=0.039)$. Given the small sample sizes we were unable to conduct a logistic regression analysis.

The lifestyle and diet of TB-diabetic and TB-IR cases and TB-controls. The proportion of TBdiabetic cases with a poor diet was lower $(10,20.4 \%)$ than among TB-controls $(32,33.0 \%)$ with TB-diabetic cases being more likely to rate their diet as good $(5,10.2 \%)$ than TB-controls $(2,2.1 \%)$ $(p=0.046)$. TB-diabetic cases were more likely to have a sedentary job $(34,69.4)$ than TB-controls $(66,68.0 \%)(p=0.029)$. With regards to IR cases, the proportion of TB-IR cases with a history of overweight during childhood was higher than among TB-controls $(4,13.8 \%$ versus $7,7.2 \%$, respectively, $\mathrm{p}=0.039)$. Given the small sample size we were unable to conduct a logistic regression analysis.

The anthropometry of male TB-diabetic and TB-IR cases and TB-controls. Male TB-diabetic cases were shorter than male TB-controls with medians of 169 and $172 \mathrm{cms}$, respectively, and a median difference of $3 \mathrm{cms}(\mathrm{p}=0.012)$. The median BMI was 24 for male TB-diabetic cases compared to 20 for male TB-controls, with a median difference of $4(\mathrm{p}=0.006)$. Male TB-diabetic cases, therefore, were more likely to be obese than male TB-controls $(7,18 \%$ versus $4,7 \%$, respectively, $p$ $=0.019)$, to have a higher median waist circumference, with a median difference of $9 \mathrm{cms}(\mathrm{p}=$ 0.002). Moreover, male TB-diabetic cases had higher median WHR than male TB-controls with median difference of 0.08 and were more likely to have high WHR $(2,5.0 \%$ versus $2,3.5 \%$, respectively) $(\mathrm{p}<0.001)$. Male TB-IR cases in turn had higher median weight than male TBcontrols (74 and $59 \mathrm{Kgs}$, respectively) with a median difference of $15 \mathrm{Kgs}(\mathrm{p}=0.028)$ and were less 
likely to have high WHR than among male TB-controls $(0,0.0 \%$ versus $2,3.5 \%$, respectively, $\mathrm{p}=$ 0.010). Given the small sample size we were unable to conduct a logistic regression analysis.

The anthropometric data of female TB-diabetic and TB-IR cases and TB-controls. The median BMI for female TB-IR cases was 24 compared to 20 for female TB-controls, with a median difference of $4(\mathrm{p}=0.036)$. On the other hand, female TB-diabetic cases had a higher weight than female TBcontrols with medians of 72 and $53 \mathrm{Kgs}$ and a median difference of $19 \mathrm{Kgs}(\mathrm{p}<0.001)$. The median BMI for female TB-diabetic cases was 30 compared to 20 for female TB-controls, with median difference of 10. Female TB-diabetic cases, therefore, were much more likely to be obese than TBcontrols $(5,56 \%$ versus $1,3 \%$, respectively), to have a higher median waist circumference with a median difference of $20 \mathrm{cms}$ and higher hip circumference than female TB-controls with a median difference of $18 \mathrm{cms}(\mathrm{p}<0.001)$. Given the small sample size we were unable to conduct a logistic regression analysis.

The metabolic characteristics TB-diabetic and TB-IR cases TB-controls. TB-diabetic cases had higher median total cholesterol than TB-controls (216 versus $183 \mathrm{mg} / \mathrm{dL}$, respectively) with a median difference of $33 \mathrm{mg} / \mathrm{dL}(\mathrm{p}<0.001)$. TB-diabetic cases also had higher median triglycerides than TB-controls (117 versus $77 \mathrm{mg} / \mathrm{dL}$, respectively) with a median difference of $40 \mathrm{mg} / \mathrm{dL}$ ( $\mathrm{p}<$ $0.001)$; and a higher proportion were classified as having high triglycerides $(5,10.2 \%$ versus 1 , $1.0 \%$, respectively, $\mathrm{p}=0.014$ ). Moreover, TB-diabetic cases had a higher median LDL Cholesterol (137 versus $112 \mathrm{mg} / \mathrm{dL}$, respectively, $\mathrm{p}=0.001$ ) and were more likely to be classified as having high LDL Cholesterol than TB-controls $(41,83.7 \%$ versus $63,64.9 \%$, respectively, $\mathrm{p}=0.018)$. Regarding cases with IR, a higher proportion of TB-IR cases had high total cholesterol than TBcontrols $(96.6 \%$ versus $79.4 \%$, respectively, $\mathrm{p}=0.043)$. Given the small sample size we were unable to conduct a logistic regression analysis. 
The symptoms of TB, X-Ray findings and TB severity score of TB-diabetic and TB-IR cases and TB-controls are shown in table 5. There were no statistical differences between the three groups, except for TB-diabetic cases being more likely to have blood in sputum than TB-controls (15, $30.6 \%$ versus $16,16.5 \%$, respectively, $\mathrm{p}=0.049)$. There were no statistical differences between the three groups regarding the TB score.

\section{Discussion}

Low- and Middle-Income Countries have witnessed a rapidly increasing incidence and prevalence of T2DM in recent decades. This increase has occurred while the global incidence of TB has declined steadily over several decades, although at a much lower rate than predicted by the WHO (19), and this condition still remains the main cause of infectious death in many countries. These two situations have resulted in both conditions increasingly being encountered as co-morbidities in the world.

The TB prevalence in the Middle East is low compared to other areas of the world as for example, by 2010 the UAE reported 6.2 cases per 100,000 population, while Saudi Arabia and Iran had reported 23 cases per 100,000 population $(20,21)$. Although these rates are relatively low in the Middle East, TB is often concentrated in specific population groups, notably migrants from Asia and Africa and the elderly.

Countries in the Middle East have also experienced major increases in T2DM. These increases are historically recent, as lifestyle patterns have mainly change since the discovery of petroleum, which in Saudi Arabia occurred in 1938, followed by an exponential increase in oil production in the 1970s. Increased wealth in Saudi Arabia was accompanied by increasing obesity, sedentary habits and T2DM. Saudi Arabia is nowadays home to a population with the highest prevalence of risk factors for T2DM such as obesity (22) and the IDF classifying Saudi Arabia as one of the ten 
countries with the highest estimated T2DM prevalence in the world. T2DM increases continued unabated in recent years, with a 2011 estimated prevalence of $16.2 \%$, which is expected to reach $20.8 \%$ by $2030(23,24)$.

The country, therefore, increasingly has patients with T2DM and populations, including migrants from places with a high prevalence of TB are, particularly, at risk of having both co-morbidities. A systematic review of the prevalence of T2DM and TB co-morbidity in Middle Eastern countries (25) reported that the prevalence of both conditions varied across the region, from as low as $4.2 \%$ in Iran to as high as $60 \%$ in Yemen $(12,26)$. African-based studies in comparison, have reported that the proportion of patients with TB that have T2DM at the time of diagnosis is generally lower, ranging from $3.4 \%$ to $16.4 \%$ of TB cases (20).

In our study, $29 \%$ of TB cases had T2DM. This high proportion is comparable to studies from areas with a high prevalence of T2DM, such as Mexico (29.3\%), Taiwan (29.5\%) and Kerala-India (44\%), but is higher than in other areas closer to the Middle East Region with low T2DM but high TB prevalence, such as Ethiopia (8.3\%) and Uganda (8.5\%) (12).

The association of T2DM and TB is not limited to LMICs. While the prevalence of TB is generally low in industrialized high-income countries, the association of TB-T2DM is generally lower, although with wide geographical variations. A cohort in Barcelona of 5849 patients with TB recruited between 2000 and 2013 reported that $4 \%$ to $7.2 \%$ had T2DM (27). This proportion was lower than in Japan, where 13.1 of patients with TB had T2DM, which was attributed to TB mostly occurring among the elderly population; and in the UK (16\%) and the USA (12\%), where obesity rates are higher than Japan and Spain (28). In these settings, T2DM still increases the risk of TB. Although this association is weaker than in low income countries. In a UK case-control study, the AORs for TB among patients with a history of T2DM was 3.8 (p < 0.05) (29). However, further case-control studies in Denmark, Australia and the USA, have reported that T2DM only modestly increased the risk of TB (20). 
In TB endemic regions there is great concern regarding the increased risk of TB that occurs due to T2DM. Bi-directional associations have been reported in the literature between hyperglycaemia and TB $(30,31)$. TB is an infection that triggers a major inflammatory process that is recognised to trigger hyperglycaemia, and it is possible the presence of inflammation has led to an over-diagnosis of T2DM. This is also the case with other conditions, such as pregnancy that can cause transient stress-related hyperglycaemia and other infections. The stress-related hyperglycaemia associated with TB could be caused by an increase in IR, which occurs through a complex interaction involving cytokines, growth hormones, catecholamine and cortisol (32-34). This IR can lead to hyperglycaemia through two pathways, the first being through hepatic glucose being overproduced via the inability to suppress gluconeogenesis, and the second is due to insulin-mediated defects in the uptake of glucose. Stress-related hyperglycaemia also leads to blood glucose levels being further increased as it enhances the inflammatory response (32). Individuals with both hyperglycaemia and TB who receive anti-TB treatment often have a substantially decreased FPG when the TB is cured. Conversely, TB can worsen a pre-existing T2DM and TB treatment outcome is poor in the presence of hyperglycaemia. Transient hyperglycaemia can be present in a significant number of individuals that already have T2DM and, therefore, this distinction is difficult at the time individuals presents to the clinic with a new episode of TB. Studies have recently indicated that after anti-TB treatment for 6 months $38 \%$ of these individuals still have hyperglycaemia, which indicates in $60 \%$ hyperglycaemia was related to the inflammatory process, while $38 \%$ had an underlying T2DM (35)

The majority of studies regarding T2DM and TB are observational, with few studies being prospective cohorts. Most cohort studies are retrospective and based on patient medical records, making it difficult to infer the directionality of the association. Although the majority of data indicate that T2DM precedes TB in a substantial number of patients, not all cases presenting with hypaerglacaemia will have the lifelong glucose abnormalities of T2DM (36). Several crosssectional studies have supported the view that T2DM increases susceptibility to TB and patients 
with TB and diabetes have a median 7 years since their T2DM diagnosis and have experienced other complications of T2DM before the development of TB (37-39).

Another possible view is that TB may increase the risk of developing T2DM, although this theory is uncommonly considered in literature at present (39). A number of studies have revealed that on the early phase of TB, IGT and/or induced hyperglycaemia occur (40). This incidence can be significant as $20-50 \%$ of individuals with IGT go on develop to T2DM, which is usually seen to occur after 3 to 5 years $(40,41)$. Establishing the real contribution of T2DM to TB is not straightforward as it can be sporadic hyperglycaemia associated to inflammation, which may reverse after TB treatment $(40,41)$ or TB may results as a result of reduced resistance to TB due to the underlying T2DM. Hyperglycaemia may also present itself as a side effect of treatment with Isoniazid and Rifampicin (42).

A Tanzanian study reported that upon enrolment, patients with TB that had glucose levels that were consistent to T2DM, newly diagnosed T2DM patients account for 50 - 80\% (based on the type of screening test) of the study group. A further study in Mwanza, Tanzania gave similar findings with $77 \%$ of individuals with T2DM were newly diagnosed T2DM cases. Studies in China and India however, have reported different results with over $50 \%$ of cases being diagnosed with T2DM before they the TB diagnosis $(35,43,44)$. The majority of the individuals that were recently diagnosed with T2DM had experienced a moderate increase in glycaemic levels. At the time of TB diagnosis, the majority of patients recently diagnosed as T2DM and pre-DM had reverted to normal glucose and were considered to have transient hyperglycaemia (35). In Iran, a third of the patients newly diagnosed to have TB had high levels of HbA1c, which after three months of anti-TB treatment had returned to normal (45). Transient hyperglycaemia is likely caused by a number of factors, which could reflect the inflammation that is caused by $\mathrm{TB}$, the predisposition of the patient and the hyperglycaemic effect that is caused by anti-TB treatment (46). This reverse causality may occur when the diagnoses of the diseases are made close together. The high occurrence of transient hyperglycaemia found in patients with TB gives rise to the notion of reverse causality between 
T2DM and TB, which also highlights the importance of further T2DM screening being implemented later into the treatment of TB. Moreover, there is also a need to address whether transient hyperglycaemia that is observed in patients diagnosed with TB would result in a greater risk of developing T2DM later in life (47).

\section{Summary of this thesis findings}

This study was carried out among patients formerly treated for TB. A high proportion of the cases were non-Saudi citizens, with the majority originating from Somalia, Yemen, Ethiopia, Pakistan, Sudan, Chad, Bangladesh, Eritrea and the Philippines. A high frequency of patients formerly treated for TB and controls without TB had T2DM, with no difference among cases and controls at the univariate analysis. Patients with T2DM, those with TB were more likely to be using insulin (20.4\%) than controls without TB (6.1\%). An important issue in the univariate analysis, however, was that cases were younger than controls. As T2DM and its risk factors increase with age, it was considered that this was likely a significant confounding factor and, therefore, its interpretation needs to be interpreted with caution, and the multivariate analysis conducted was controlled by age. The results of the final multivariable backward stepwise logistic regression including T2DM indicate that cases were more likely to be non-Saudis, to have T2DM and that they had a poorer diet than controls. Cases were more likely to eat fast foods $1-2$ times/week or $2-3$ times/month, less likely to be obese, to have higher total cholesterol and a lower level of risky HDL Cholesterol than controls. On the other hand, when we replaced variables for T2DM and replaced them with the more comprehensive definition of metabolic syndrome, the multivariable backward stepwise logistic regression results indicated cases were more likely to be non-Saudis and had a poorer diet than controls. Cases were also less likely to be obese and to have higher total cholesterol than controls. 
TB-T2DM and TB-IR cases were also compared with TB-controls to identify risk factors for T2DM or IR. Both male and female patients with T2DM had higher BMI and were much more likely to be obese, had higher cholesterol and triglycerides. Moreover, patients with T2DM had higher LDL cholesterol and more likely to have experienced haemoptysis than patients without T2DM. Female cases with T2DM were more likely to have experienced gestational diabetes. Similarly, the comparison of TB-IR and TB-controls indicated patients with IR were more likely to have experienced heart problems, to have been overweight during childhood, total cholesterol and females had higher median BMI. These characteristics replicate well established risk factors for both T2DM and IR among the general population and their association to TB is still unclear.

Although many studies have reported the increased prevalence of T2DM among patients with TB at the time of making the diagnosis of $\mathrm{TB}$, there are very few studies examining whether patients formerly treated for TB continue to have T2DM several months after treatment when the inflammatory processes associated with TB have subsided. Patients with TB often have IFG, for example, in West Africa 1.9\% of patients with TB had T2DM and 5\% had IFG (26). In Sri Lanka, $7.1 \%$ of patients with TB had T2DM and $20 \%$ had IFG (27). Although IFG could reflect a predisposition to T2DM, IFG is also likely due to the pro-inflammatory responses to TB, the effect of medications or the 'stress/transient hyperglycaemia' phenomenon (9).

We measured IR with the HOMA2-IR method which describes the balance of insulin levels in the context of fasting glucose conditions. The HOMA-IR is extensively used as a regular measurement tool in clinical practice and epidemiological research and is one of the more popular fasting indices (48). Although the hyperinsulinaemic-euglycaemic clamp technique is still considered the goldstandard for IR diagnosis and is still the diagnostic tool of preference in some clinical settings, it was not a viable option in this study, as study subjects were investigated in out-patient departments and the tool is time consuming. One significant disadvantage of the HOMA-IR is that it does not have a standardized limit to categorize individuals that have IR. Prior studies have indicated that IR appears between HOMA-IR levels of 1.55 - 3.8 (49), which is a large range. In this study we used a 
cut-off $\geq 2$, as this is the cut-off used in most publications. Using this cut-off, in participants without T2DM, the prevalence of IR among euglycaemic patients with former TB was $22 \%$. This prevalence rates fall between the $20 \%$ to $40 \%$ IR rates reported in the general population of Saudi Arabia (48) and, therefore, we found no evidence that IR is more or less frequent among patients who formerly had TB.

A prospective cohort study in patients with TB in South Africa suggested a gradual decrease in IR after initiation of TB treatment. Moreover, when confounding variables were considered, the study reported that HOMA-IR was not associated with the diagnosis of TB. These findings thus suggest IR in a significant proportion of the patients could be due to the 'stress/transient' hyperglycaemia that is present early in the diagnosis of TB and that is eventually resolved once treatment for TB progresses (50-52).

IR has long been linked to the ageing process, $(53,54)$, as it is generally linked to an increase in fat mass and body weight, particularly in the centre of the body (55), along with a greater prevalence of chronic lifestyle-associated diseases such as metabolic syndrome (56). Generally, an increased anthropometrical measurement gives a greater risk of IR; (57) with a positive correlation between the waist-to-hip ratio (58) and the BMI (59).

Studies in the past have also suggested that undernutrition does not only occur in TB patients due to the disease itself, but rather to a number of other factors such as reduced health-seeking behaviour, extreme poverty and food insecurity $(60,61)$. Indeed, several studies have suggested that epigenetics may play a critical role in the Middle East populations, explaining the higher prevalence of both T2DM and IR. A recent study suggested epigenetic changes in these populations share many of the characteristics reported in European populations, but that some novel mechanisms are unique to Arab populations; suggesting that the underlying mechanisms in the region might depend on their genetic background and local environmental pressures (62). We have written in reverse causality the directionality of association between T2DM and TB. 


\section{Recommendations for clinical practice}

Clinical practice recommendations include the application of bi-directional (TB and T2DM) screening in healthcare facilities along with the monitoring patients with TB upon commencing treatment for the presence of hyperglycaemia. Although not considered in this thesis, patients with T2DM have poorer response to TB treatment and newly diagnosed patients with TB and T2DM should be followed closely to monitor treatment response. Patients with clinical symptoms of T2DM, biochemical or anthropometrical markers should also be referred for nutritional support in a timely manner, preferably at the intensive treatment phase of TB.

Future avenues of research include assessing how IR prevalence changes in cohort studies to describe changes in T2DM and IR prevalence and to identify individuals with overt T2DM that require long term management. Furthermore, patients with established T2DM could be compared to patients who have transient hyperglycaemia to explore how to differentiate both conditions at the time of TB diagnosis. Finally, patients with transient hyperglycaemia may go on to develop over T2DM, as observed among women with gestational diabetes, and should be followed over time.

\section{Limitations}

The study has significant limitations. These include the lack of standardised HOMA-IR reference values, along with potential reliability issues surrounding the measurement of fasting insulin. Although the former are limitations shared with other studies, the latter was due to logistical constrains when conducting the study, as it was not possible to obtain external quality assurance to confirm insulin measurements were correct. Although laboratories in Saudi Arabia undergo rigorous quality control procedures, it would have been ideal to replicate a subset of tests in a second quality-assured laboratory to confirm the reliability of the measurements. 
There are also limitations during the study implementation, as one city had very few patients with TB and patients were recruited in two cities 300 kilometres apart, which limited the supervision to alternative weeks. There may have been compliance issues with the requirement of participants' interview, sample collection and transport which the $\mathrm{PhD}$ candidate could not oversee all the time.

A further limitation is that patients were enrolled several months after the initial TB diagnosis, which could have generated recall bias and lost to follow up, especially among non-Saudi patients who may have left the country. Ideally, patients should have been enrolled at the time of their TB diagnosis for prospective follow up as cohorts with and without T2DM or IR.

With regards to selection biases and methodological limitations, it was clear the choice of controls was not adequate, as controls were older than TB cases and very different to patients with CHD. These substantial different likely precluded correcting these differences by the use of logistic regression s, and this is acknowledged as a major limitation of this thesis.

Lastly, the sample size of the study should have been bigger. Although the data collected met the original samples size estimations, we did not expect to find the high prevalence of T2DM and IR among controls and, therefore, the study is likely to be underpowered. The high prevalence of T2DM in cases with TB resulted in a small sample size of the group with TB and IR and, therefore, we were unable to conduct a logistic regression analysis. It is thus likely the study did not identify metabolic anomalies that might be present at a low frequency among patient populations with a lower prevalence of T2DM among the general population.

\section{Strengths}

The study provided baseline information, which will help Saudi Arabia to strengthen the TB control programme and increase the knowledge toward exploring the prevalence of euglycaemic IR among TB patients, its role as a risk factor for TB and the severity of clinical presentation, which are 
poorly established in Saudi Arabia. A further strength is the high response rate, as most eligible patients accepted to participate. Further, a team of trained interviewers interviewed the participants at a mutually convenient location either in their own homes or the healthcare facilities to meet participant satisfaction. Despite the hot weather, blood sample transportation was within two hours of collection by a reporter using a box and container with ice under safety precautions and samples were processed in fully accredited, a high-quality laboratory in King Fahad Hospital.

The availability of data and adequacy for testing the hypothesis is integral to the validity of a retrospective case-control study. A key strength of the data collection was using the ministry of health TB databases to complete missing information and to verify data collected at the time of the interview that referred to the time of TB diagnosis.

\section{Conclusion}

The results of this study suggest that the frequency of T2DM and IR in patients with TB was very high with more than a quarter of patients having T2DM. However, the frequency of IR was not higher among patients with TB than among individuals without TB, reflecting the very high prevalence of IR among the general population. Patients with a history of TB had lower HDL cholesterol; were more likely to be prediabetic and to be non-Saudi. Patients with TB and T2DM or IR shared the same characteristics as patients with T2DM and IR without TB. Patients with IR were more likely to have experienced CVDs and to be obese or overweight and report poor diet habits, while the lack of exercise and active lifestyles were nearly non-existent. There is a need for early identification programs, in particular within vulnerable and young populations to enable IR reversal and to prevent future complications associated with IR and T2DM. 


\section{References:}

1. Type 2 Diabetes Mellitus A Review of Current Trends.

2. Islam SMS, Purnat TD, Phuong NTA, Mwingira U, Schacht K, Fröschl G. Non- Communicable Diseases (NCDs) in developing countries: a symposium report. Globalization and health. $2014 ; 10(1): 81$. 3. Organization WH. Definition and diagnosis of diabetes mellitus and intermediate hyperglycemia 2006 [cited 2016 22/02/2016]. Available from: http://www.who.int/diabetes/publications/Definition\%20and\%20diagnosis\%20of\%20diabetes_new.pdf 4. Diabetes atlas: International Diabetes Federation; 2013 [cited 201625 Februrary]. 6th:[Available from: http://www.idf.org/sites/default/files/EN_6E_Atlas_Full_0.pdf.

5. Global tuberculosis report: World Health Organization. ; 2014 [cited 201625 Februrary]. Available from: http://apps.who.int/iris/bitstream/10665/137094/1/9789241564809_eng.pdf.

6. Ogbera AO, Kapur A, Chinenye S, Fasanmade O, Uloko A, Odeyemi K. Undiagnosed diabetes mellitus in tuberculosis: A Lagos report. Indian Journal of Endocrinology and Metabolism. 2014;18(4):4759.

7. Wang H-T, Zhang J, Ji L-C, You S-H, Bai Y, Dai W, et al. Frequency of tuberculosis among diabetic patients in the People's Republic of China. Therapeutics and Clinical Risk Management. 2014;10:45-9.

8. Alisjahbana B, Sahiratmadja E, Nelwan EJ, Purwa AM, Ahmad Y, Ottenhoff TH, et al. The effect of type 2 diabetes mellitus on the presentation and treatment response of pulmonary tuberculosis. Clin Infect Dis. 2007;45(4):428-35.

9. Chang J-T, Dou H-Y, Yen C-L, Wu Y-H, Huang R-M, Lin H-J, et al. Effect of Type 2 Diabetes Mellitus on the Clinical Severity and Treatment Outcome in Patients With Pulmonary Tuberculosis: A Potential Role in the Emergence of Multidrug-resistance. Journal of the Formosan Medical Association. 2011;110(6):372-81.

10. Jimenez-Corona ME, Cruz-Hervert LP, Garcia-Garcia L, Ferreyra-Reyes L, Delgado-Sanchez G, Bobadilla-Del-Valle M, et al. Association of diabetes and tuberculosis: impact on treatment and posttreatment outcomes. Thorax. 2013;68(3):214-20. 
11. Balakrishnan S, Vijayan S, Nair S, Subramoniapillai J, Mrithyunjayan S, Wilson N, et al. High diabetes prevalence among tuberculosis cases in Kerala, India. PLoS One. 2012;7(10):e46502.

12. Workneh MH, Bjune GA, Yimer SA. Prevalence and Associated Factors of Diabetes Mellitus among Tuberculosis Patients in South-Eastern Amhara Region, Ethiopia: A Cross Sectional Study. PLoS One. 2016;11(1):e0147621.

13. ReyPineda G D. Type 2 Diabetes Mellitus as a Risk Factor for Tuberculosis. Mycobacterial Diseases. 2014;04(02).

14. Diabetes and tuberculosis old associates posing a renewed public health challenge.pdf.

15. Kapur A, Harries AD. The double burden of diabetes and tuberculosis - public health implications. Diabetes Res Clin Pract. 2013;101(1):10-9.

16. Bukhary ZA. Rediscovering the Association Between Tuberculosis and Diabetes Mellitus: A Perspective. Journal of Taibah University Medical Sciences. 2008;3(1):1-6.

17. WHO. Diabetes Geneva: World Health Organisation; 2018 [Available from: https://www.who.int/news-room/fact-sheets/detail/diabetes.

18. Bermúdez V, Rojas J, Martínez MS, Apruzzese V, Chávez-Castillo M, Gonzalez R, et al. Epidemiologic behavior and estimation of an optimal cut-off point for homeostasis model assessment-2 insulin resistance: a report from a Venezuelan population. International Scholarly Research Notices. 2014;2014.

19. Harries A, Satyanarayana S, Kumar A, Nagaraja S, Isaakidis P, Malhotra S, et al. Epidemiology and interaction of diabetes mellitus and tuberculosis and challenges for care: a review. Public health action. 2013;3(1):3-9.

20. Zheng CL, Hu MH, Gao F. Diabetes and pulmonary tuberculosis: a global overview with special focus on the situation in Asian countries with high TB-DM burden. Global Health Action. 2017;10:1-11.

21. Tuberculosis in the Middle East [Internet]. The Lancet Middle East Edition. 2012 [cited 2012].

Available from: http://www.thelancet.com/pb/assets/raw/Lancet/global-health/middleeast/Dec12_MiddleEastEd.pdf.

22. Kaplan SA. Re: National, regional, and global trends in body-mass index since 1980: systematic analysis of health examination surveys and epidemiological studies with 960 country-years and 9.1 million participants. J Urol. 2011;186(5):1982-3.

23. Al-Quwaidhi AJ, Pearce MS, Sobngwi E, Critchley JA, O’Flaherty M. Comparison of type 2 diabetes prevalence estimates in Saudi Arabia from a validated Markov model against the International Diabetes Federation and other modelling studies. Diabetes Research and Clinical Practice. 2014;103(3):496503.

24. Whiting DR, Guariguata L, Weil C, Shaw J. IDF Diabetes Atlas: Global estimates of the prevalence of diabetes for 2011 and 2030. Diabetes Research and Clinical Practice. 2011;94(3):311-21.

25. Alkabab YM, Al-Abdely HM, Heysell SK. Diabetes-related tuberculosis in the Middle East: an urgent need for regional research. International Journal of Infectious Diseases. 2015;40:64-70.

26. Alkabab YM, Al-Abdely HM, Heysell SK. Diabetes-related tuberculosis in the Middle East: an urgent need for regional research. Int J Infect Dis. 2015;40:64-70.

27. Moreno-Martinez A, Casals M, Orcau A, Gorrindo P, Masdeu E, Cayla JA, et al. Factors associated with diabetes mellitus among adults with tuberculosis in a large European city, 2000-2013. International Journal of Tuberculosis and Lung Disease. 2015;19(12):1507-12.

28. Uchimura K, Ngamvithayapong-Yanai J, Kawatsu L, Ohkado A, Yoshiyama T, Shimouchi A, et al. Characteristics and treatment outcomes of tuberculosis cases by risk groups, Japan, 2007-2010. Western Pacific Surveillance and Response. 2013;4(1).

29. Jick SS, Lieberman ES, Rahman MU, Choi HK. Glucocorticoid use, other associated factors, and the risk of tuberculosis. Arthritis \& Rheumatism-Arthritis Care \& Research. 2006;55(1):19-26.

30. Jeon CY, Murray MB. Diabetes mellitus increases the risk of active tuberculosis: a systematic review of 13 observational studies. PLoS medicine. 2008;5(7):e152.

31. Jiménez-Corona ME, Cruz-Hervert LP, García-García L, Ferreyra-Reyes L, Delgado-Sánchez G, Bobadilla-del-Valle M, et al. Association of diabetes and tuberculosis: impact on treatment and posttreatment outcomes. Thorax. 2013;68(3):214-20.

32. Aftab H, Christensen DL, Ambreen A, Jamil M, Garred P, Petersen JH, et al. Tuberculosis-Related Diabetes: Is It Reversible after Complete Treatment? The American journal of tropical medicine and hygiene. 2017;97(4):1099-102.

33. Chrousos GP. The hypothalamic-pituitary-adrenal axis and immune-mediated inflammation. N Engl J Med. 1995;332(20):1351-63. 
34. Barth E, Albuszies G, Baumgart K, Matejovic M, Wachter U, Vogt J, et al. Glucose metabolism and catecholamines. Critical care medicine. 2007;35(9):S508-S18.

35. Boillat-Blanco N, Ramaiya KL, Mganga M, Minja LT, Bovet P, Schindler C, et al. Transient hyperglycemia in patients with tuberculosis in Tanzania: implications for diabetes screening algorithms. The Journal of infectious diseases. 2015;213(7):1163-72.

36. Kuo MC, Lin SH, Lin CH, Mao IC, Chang SJ, Hsieh MC. Type 2 Diabetes : An Independent Risk

Factor for Tuberculosis: A Nationwide Population-Based Study. Plos One. 2013;8(11).

37. Restrepo BI, Camerlin AJ, Rahbar MH, Wang WW, Restrepo MA, Zarate I, et al. Cross-sectional assessment reveals high diabetes prevalence among newly-diagnosed tuberculosis cases. Bulletin of the World Health Organization. 2011;89(5):352-9.

38. Abdelbary BE, Garcia-Viveros M, Ramirez-Oropesa H, Rahbar MH, Restrepo BI. Tuberculosisdiabetes epidemiology in the border and non-border regions of Tamaulipas, Mexico. Tuberculosis.

2016;101:S124-S34.

39. Stevenson CR, Critchley JA, Forouhi NG, Roglic G, Williams BG, Dye C, et al. Diabetes and the risk of tuberculosis: a neglected threat to public health? Chronic Illness. 2007;3(3):228-45.

40. Bacakoğlu F, Başoğlu ÖK, Çok G, Sayıner A, Ateş M. Pulmonary tuberculosis in patients with diabetes mellitus. Respiration. 2001;68(6):595-600.

41. Oluboyo PO, Erasmus RT. The significance of glucose-intolerance in pulmonary tuberculosis. Tubercle. 1990;71(2):135-8.

42. Niemi M, Backman JT, Neuvonen M, Neuvonen PJ, Kivisto KT. Effects of rifampin on the pharmacokinetics and pharmacodynamics of glyburide and glipizide. Clinical Pharmacology \& Therapeutics. 2001;69(6):400-6.

43. Viswanathan V, Kumpatla S, Aravindalochanan V, Rajan R, Chinnasamy C, Srinivasan R, et al. Prevalence of diabetes and pre-diabetes and associated risk factors among tuberculosis patients in India. PloS one. 2012;7(7):e41367.

44. Wang Q, Ma A, Han X, Zhao S, Cai J, Ma Y, et al. Prevalence of type 2 diabetes among newly detected pulmonary tuberculosis patients in China: a community based cohort study. PloS one.

2013;8(12):e82660.

45. Tabarsi P, Baghaei P, Marjani M, Vollmer WM, Masjedi M-R, Harries AD. Changes in glycosylated haemoglobin and treatment outcomes in patients with tuberculosis in Iran: a cohort study. Journal of Diabetes \& Metabolic Disorders. 2014;13(1):123.

46. Dungan KM, Braithwaite SS, Preiser J-C. Stress hyperglycaemia. The Lancet. 2009;373(9677):1798-807.

47. Chen R, Mias GI, Li-Pook-Than J, Jiang L, Lam HY, Chen R, et al. Personal omics profiling reveals dynamic molecular and medical phenotypes. Cell. 2012;148(6):1293-307.

48. Philips L, Visser J, Nel D, Blaauw R. The association between tuberculosis and the development of insulin resistance in adults with pulmonary tuberculosis in the Western sub-district of the Cape Metropole region, South Africa: a combined cross-sectional, cohort study. BMC infectious diseases. 2017;17(1):570. 49. Al Qarni AA, Joatar FE, Das N, Awad M, Eltayeb M, Al-Zubair AG, et al. Association of Plasma Ghrelin Levels with Insulin Resistance in Type 2 Diabetes Mellitus among Saudi Subjects. Endocrinol Metab (Seoul). 2017;32(2):230-40.

50. Kapur A, Harries AD. The double burden of diabetes and tuberculosis-public health implications. Diabetes research and clinical practice. 2013;101(1):10-9.

51. Jawad F, Shem A, Memon R, Ansari G. Glucose intolerance in pulmonary tuberculosis. JOURNALPAKISTAN MEDICAL ASSOCIATION. 1995;45:237-8.

52. Singh M, Biswas S, Shah A. Impaired glucose tolerance in active pulmonary tuberculosis. Indian J Tuberc. 1984;31(3):118-21.

53. Katz A, Nambi SS, Mather K, Baron AD, Follmann DA, Sullivan G, et al. Quantitative insulin sensitivity check index: a simple, accurate method for assessing insulin sensitivity in humans. The Journal of Clinical Endocrinology \& Metabolism. 2000;85(7):2402-10.

54. DeFronzo RA, Tobin JD, Andres R. Glucose clamp technique: a method for quantifying insulin secretion and resistance. American Journal of Physiology-Endocrinology And Metabolism. 1979;237(3):E214.

55. Ryan AS. Insulin resistance with aging. Sports medicine. 2000;30(5):327-46.

56. Ford ES. Prevalence of the metabolic syndrome defined by the International Diabetes Federation among adults in the US. Diabetes Care. 2005;28(11):2745-9. 
57. Simarro MR, Carbayo JH, Massó JO, Artigao LR, Carrión LV, Divisón JG, et al. Association of insulin resistance to different anthropometric measures and cardiovascular risk factors in a non-diabetic population. Endocrinologia y nutricion: organo de la Sociedad Espanola de Endocrinologia y Nutricion. 2011;58(9):464-71.

58. Gayoso-Diz P, Otero-Gonzalez A, Rodriguez-Alvarez MX, Gude F, Cadarso-Suarez C, García F, et al. Insulin resistance index (HOMA-IR) levels in a general adult population: curves percentile by gender and age. The EPIRCE study. Diabetes research and clinical practice. 2011;94(1):146-55.

59. Esteghamati A, Khalilzadeh O, Anvari M, Ahadi MS, Abbasi M, Rashidi A. Metabolic syndrome and insulin resistance significantly correlate with body mass index. Archives of medical research. 2008;39(8):803-8.

60. Dodor E. Evaluation of nutritional status of new tuberculosis patients at the effia-nkwanta regional hospital. Ghana medical journal. 2008;42(1):22.

61. Villamor E, Saathoff E, Mugusi F, Bosch R, Urassa W, Fawzi W. Wasting and body composition of adults with pulmonary tuberculosis in relation to HIV-1 coinfection, socioeconomic status, and severity of tuberculosis. European journal of clinical nutrition. 2006;60(2):163.

62. Al Muftah WA, Al-Shafai M, Zaghlool SB, Visconti A, Tsai P-C, Kumar P, et al. Epigenetic associations of type 2 diabetes and BMI in an Arab population. Clinical epigenetics. 2016;8(1):13. 
Figure 1. Flow chart of TB study:

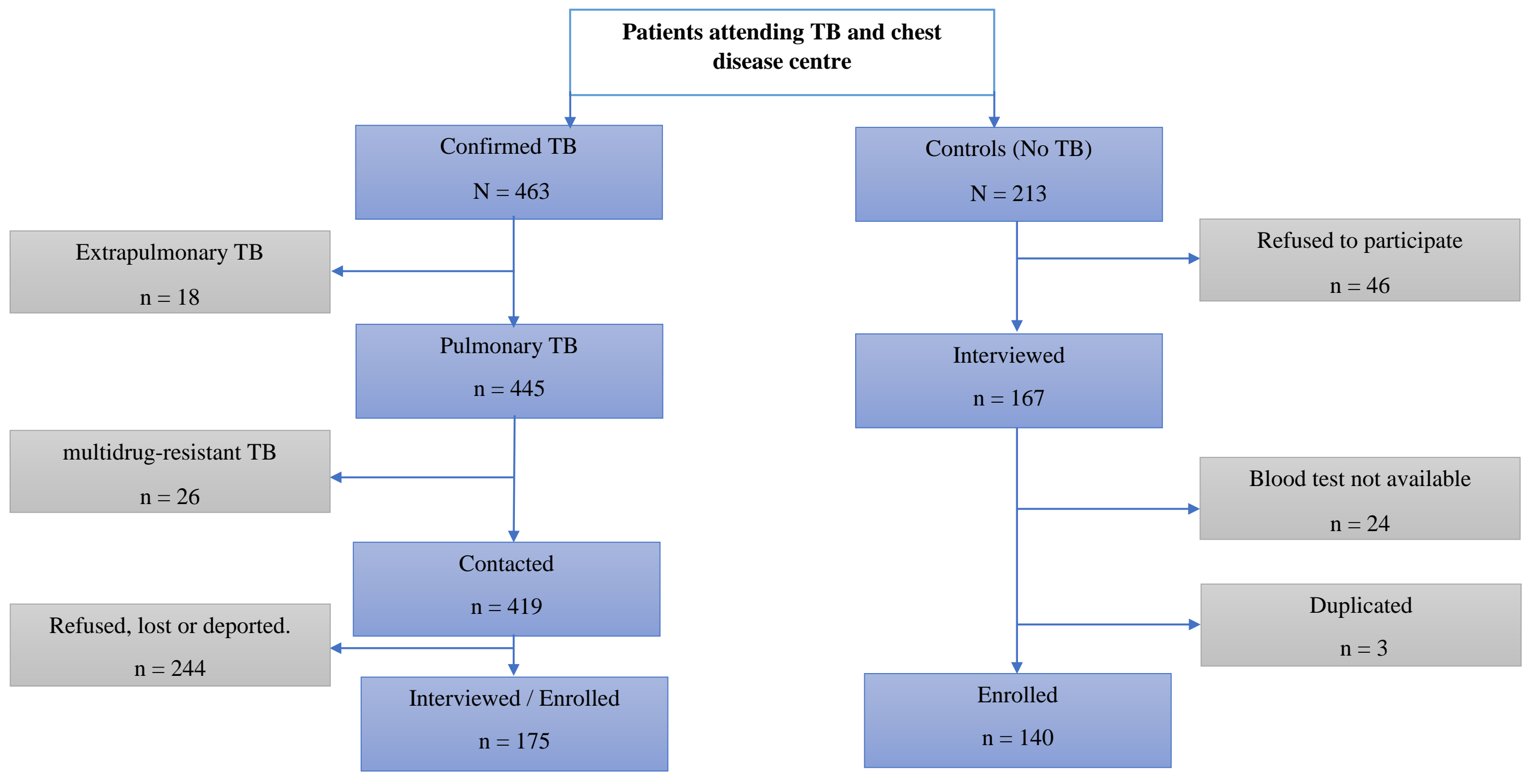


Table 1. Demographic characteristics of cases and controls.

\begin{tabular}{|c|c|c|c|c|}
\hline \multicolumn{2}{|c|}{$\begin{array}{l}\text { Demographic characteristics } \\
N=315\end{array}$} & \multirow{2}{*}{$\begin{array}{c}\text { Cases } \\
\mathbf{n}=\mathbf{1 7 5}(\%) \\
111(63.4)\end{array}$} & \multirow{2}{*}{$\begin{array}{c}\text { Controls } \\
\mathbf{n}=\mathbf{1 4 0}(\%) \\
83(59.3)\end{array}$} & $p$ \\
\hline Sex & Male & & & \multirow{2}{*}{0.453} \\
\hline & Female & $64(36.6)$ & $57(40.7)$ & \\
\hline \multirow[t]{2}{*}{ Age } & Range & $18-78$ & $18-76$ & \multirow{2}{*}{$<0.001$} \\
\hline & Median (IQR) & $33(25-45)$ & $40(28-53)$ & \\
\hline \multirow[t]{4}{*}{ Age group } & $18-29$ & $71(40.6)$ & $39(27.9)$ & \multirow{4}{*}{$0.0 \cdot r$} \\
\hline & $30-39$ & $45(25.7)$ & $23(16.4)$ & \\
\hline & $40-49$ & $22(12.6)$ & $29(20.7)$ & \\
\hline & $\geq 50$ & $37(21.1)$ & $49(35.0)$ & \\
\hline \multirow[t]{2}{*}{ Ethnicity } & Saudi & $37(21.1)$ & $73(52.1)$ & \multirow{2}{*}{$<0.001$} \\
\hline & Non-Saudi & $138(78.9)$ & $67(47.9)$ & \\
\hline
\end{tabular}

Table 2. T2DM and IR cases among cases and controls.

\begin{tabular}{|l|c|c|c|c|c|}
\hline $\begin{array}{l}\text { Study population } \\
\mathbf{N}=\mathbf{3 1 5}\end{array}$ & $\begin{array}{c}\text { Cases } \\
\mathbf{n = 1 7 5 ( \% )}\end{array}$ & $\mathbf{9 5 \%} \mathbf{C I}$ & $\begin{array}{c}\text { Controls } \\
\mathbf{n = 1 4 0}(\boldsymbol{\%})\end{array}$ & $\mathbf{9 5 \%} \mathbf{C I}$ & $\boldsymbol{p}$ \\
\hline T2DM participants & $51(29.0)$ & $22.5-36.5$ & $39(27.9)$ & $20.6-36.1$ & 0.801 \\
\hline T2DM using insulin & $10(19.6)$ & $9.8-33.1$ & $2(5.1)$ & $0.6-17.3$ & 0.061 \\
\hline T2DM not using insulin & $41(80.4)$ & $66.9-90.2$ & $37(94.9)$ & $82.7-99.4$ & 0.801 \\
\hline Normoglycemic participants & $124(71.0)$ & $63.5-77.5$ & $101(72.1)$ & $63.9-79.4$ & 0.485 \\
\hline Normoglycemic with IR & $27(22.0)$ & $14.9-30.1$ & $26(25.7)$ & $17.6-35.4$ & 6 \\
\hline Normoglycemic without IR & $97(78.0)$ & $69.9-85.1$ & $75(74.3)$ & $64.6-82.4$ & $40.0-57.2$ \\
\hline Participants with MS & $45(25.7)$ & $19.4-32.9$ & $68(48.6)$ & 40.0 & $<0.001$ \\
\hline Participants without MS & $130(74.3)$ & $67.1-80.6$ & $72(51.4)$ & $42.8-60.0$ & \\
\hline
\end{tabular}


Figure 2. Diabetes status of cases and controls

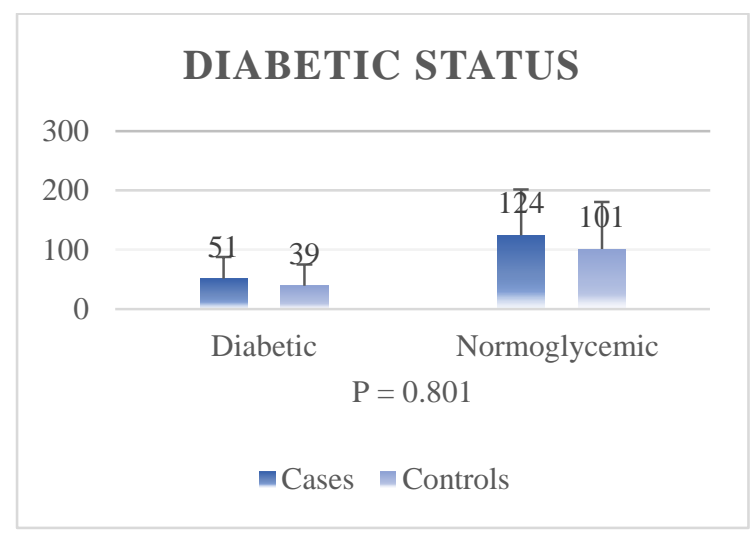

Figure 3. Insulin use among cases and controls

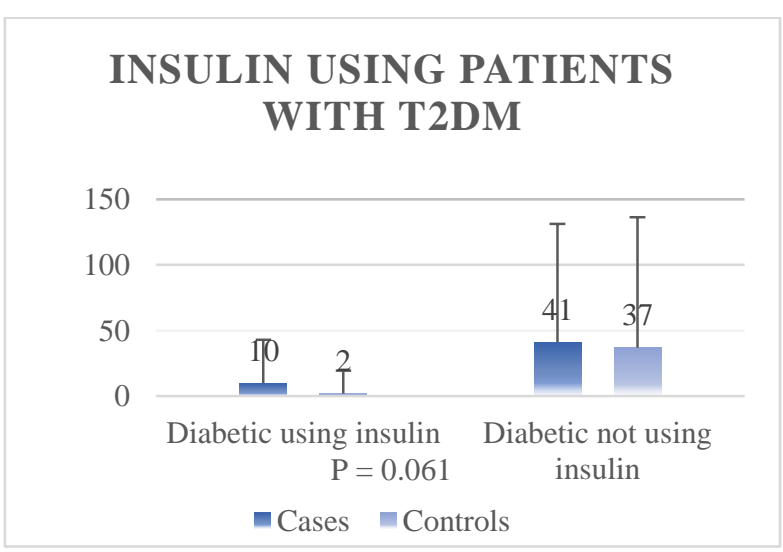

Figure 4. IR status of normoglycemic cases and controls

\begin{tabular}{|c|c|}
\hline \multirow{5}{*}{$\begin{array}{r}200 \\
150 \\
100 \\
50 \\
0\end{array}$} & $\begin{array}{c}\text { IR AMONG } \\
\text { NORMOGLYCEMIC }\end{array}$ \\
\hline & $97 \quad 75$ \\
\hline & $\begin{array}{c}\text { Normoglycemic with IR Normoglycemic without } \\
\text { IR }\end{array}$ \\
\hline & $P=0.485$ \\
\hline & -Cases Controls \\
\hline
\end{tabular}


Table 3. Logistic regression model for factors predicting TB including T2DM.

\begin{tabular}{|c|c|c|c|}
\hline \multirow{2}{*}{\multicolumn{2}{|c|}{$\begin{array}{l}\text { Variables } \\
N=315\end{array}$}} & \multicolumn{2}{|l|}{ Multivariate } \\
\hline & & \multirow{2}{*}{$\begin{array}{r}\mathrm{AOR} *(95 \% \mathrm{CI}) \\
1\end{array}$} & \multirow[t]{2}{*}{$p$} \\
\hline \multirow[t]{2}{*}{ Ethnicity } & Saudi & & \\
\hline & Non-Saudi & $3.565(1.83-6.97)$ & $<0.001$ \\
\hline \multirow[t]{2}{*}{ T2DM } & No & \multicolumn{2}{|l|}{1} \\
\hline & Yes & $2.950(1.31-6.62)$ & 0.009 \\
\hline \multirow{3}{*}{$\begin{array}{l}\text { Participant self- } \\
\text { rating the diet } \\
\text { quality }\end{array}$} & Good & \multicolumn{2}{|l|}{$\begin{array}{ll}1 \\
\end{array}$} \\
\hline & Fair & $12.944(5.60-29.93)$ & $<0.001$ \\
\hline & Poor & $33.625(10.75-105.20)$ & $<0.001$ \\
\hline \multirow{5}{*}{$\begin{array}{l}\text { Fast food } \\
\text { consumption }\end{array}$} & $0-1 /$ Month & \multicolumn{2}{|l|}{1} \\
\hline & 2 - 3/Month & $2.655(1.00-7.04)$ & 0.050 \\
\hline & 1 - 2/Week & $2.468(0.03-5.93)$ & 0.043 \\
\hline & $3-4 /$ Week & $0.490(0.18-1.34)$ & 0.166 \\
\hline & $\geq 5 /$ Week & $1.027(0.36-2.91)$ & 0.960 \\
\hline \multirow[t]{4}{*}{ BMI* groups } & $\leq 18.4$ & \multicolumn{2}{|l|}{1} \\
\hline & $18.5-24.9$ & $0.627(0.23-1.73)$ & 0.367 \\
\hline & $25-29.9$ & $0.307(0.09-1.00)$ & 0.050 \\
\hline & $\geq 30$ & $0.190(0.05-0.70)$ & 0.012 \\
\hline \multirow{2}{*}{$\begin{array}{l}\text { Total cholesterol } \\
(\mathrm{mg} / \mathrm{dL})\end{array}$} & $<150$ & \multicolumn{2}{|l|}{1} \\
\hline & $\geq 150$ & $3.035(1.34-6.87)$ & 0.008 \\
\hline \multirow{2}{*}{$\begin{array}{l}\text { HDL* Cholesterol } \\
(\mathrm{mg} / \mathrm{dL})\end{array}$} & Optimal & \multicolumn{2}{|l|}{1} \\
\hline & Risky & $0.400(0.21-0.76)$ & 0.005 \\
\hline \multicolumn{2}{|l|}{ Constant } & 0.090 & 0.005 \\
\hline
\end{tabular}

*AOR $=$ Adjusted Odds Ratio, $*$ BMI $=$ Body Mass index, $*$ FPG $=$ Fasting plasma glucose,$*$ HbA1c $=$ Glycated haemoglobin, $*$ HDL = High-density lipoprotein.

Table 4. Logistic regression model for factors predicting TB including metabolic syndrome.

\begin{tabular}{|c|c|c|c|}
\hline \multirow{2}{*}{\multicolumn{2}{|c|}{$\begin{array}{c}\text { Variables } \\
\mathrm{N}=315\end{array}$}} & \multicolumn{2}{|c|}{ Multivariate } \\
\hline & & AOR* $(95 \%$ CI $)$ & $p$ \\
\hline \multirow[t]{2}{*}{ Ethnicity } & Saudi & \multicolumn{2}{|l|}{1} \\
\hline & Non-Saudi & $3.448(1.81-6.58)$ & $<0.001$ \\
\hline \multirow{2}{*}{$\begin{array}{l}\text { Metabolic } \\
\text { syndrome }\end{array}$} & No & \multicolumn{2}{|l|}{1} \\
\hline & Yes & $0.881(0.42-1.86)$ & 0.741 \\
\hline \multirow{3}{*}{$\begin{array}{l}\text { Participant self- } \\
\text { rating the diet } \\
\text { quality }\end{array}$} & Good & \multicolumn{2}{|l|}{1} \\
\hline & Fair & $12.893(5.71-29.11)$ & $<0.001$ \\
\hline & Poor & $30.197(10.10-90.29)$ & $<0.001$ \\
\hline BMI* groups & $\leq 18.4$ & \multicolumn{2}{|l|}{1} \\
\hline
\end{tabular}




\begin{tabular}{|l|l|c|c|}
\hline & $18.5-24.9$ & $0.632(0.24-1.70)$ & 0.362 \\
\cline { 2 - 4 } & $25-29.9$ & $0.320(0.10-1.02)$ & 0.054 \\
\cline { 2 - 4 } & $\geq 30$ & $0.224(0.06-0.81)$ & $\mathbf{0 . 0 2 2}$ \\
\hline $\begin{array}{l}\text { Total cholesterol } \\
\text { (mg/dL) }\end{array}$ & $<150$ & $\mathbf{1}$ & $\mathbf{0 . 0 0 2}$ \\
\hline Constant & $\geq 150$ & $3.382(1.55-7.40)$ & 0.001 \\
\hline
\end{tabular}

Table 5. TB symptoms, X-Ray and scoring of TB-IR cases, TB-diabetic cases and TB-controls

\begin{tabular}{|c|c|c|c|c|c|}
\hline $\begin{array}{l}\text { TB symptoms, } x \text {-Ray and } \\
\text { scoring } \\
N=175\end{array}$ & $\begin{array}{c}\text { TB-Controls } \\
\mathbf{n}=97(\%)\end{array}$ & $\begin{array}{c}\text { TB-IR } \\
\text { n = 29 } \\
(\%)\end{array}$ & $\boldsymbol{P}$ & $\begin{array}{c}\text { TB- } \\
\text { T2DM } \\
\text { n= 49 }(\%)\end{array}$ & $P$ \\
\hline Sputum with blood & $16(16.5)$ & $6(20.7)$ & 0.602 & $15(30.6)$ & 0.049 \\
\hline
\end{tabular}

